\title{
Computing Fifth Geometric-Arithmetic Index for Circumcoronene series of benzenoid $\boldsymbol{H}_{k}$ \\ Mohammad Reza Farahani \\ Department of Applied Mathematics, Iran University of Science and Technology (IUST), \\ Narmak, Tehran 16844, Iran \\ Mr_Farahani@Mathdep.iust.ac.ir
}

\section{ABSTRACT}

Let $G=(V ; E)$ be a simple connected graph. The sets of vertices and edges of $G$ are denoted by $V=V(G)$ and $E=E(G)$, respectively. The geometric-arithmetic index is a topological index was introduced by Vukicevic and Furtula in 2009 and defined as $G A \mathrm{G}=\sum_{\mathrm{uv} \in \mathrm{E}} \frac{2 \sqrt{\mathrm{d}_{u} \mathrm{~d}_{v}}}{\mathrm{~d}_{u}+\mathrm{d}_{v}}$ in which degree of vertex $u$ denoted by $d_{G}(u)$ (or $d_{u}$ for short). In 2011, A. Graovac et al defined a new version of $G A$ index as $G A_{5} G=\sum_{u v \in E(G)} \frac{2 \sqrt{S_{v} S_{u}}}{S_{v}+S_{u}}$ where $S_{v}=\sum_{u v \in E(G)} d_{u}$. The goal of this paper is to compute the fifth geometric-arithmetic index for "Circumcoronene series of benzenoid $H_{k}(k \geq 1)$ ".

\section{Indexing terms/Keywords}

Molecular graph; Circumcoronene Series of Benzenoid, Geometric-Arithmetic Index;

\section{SUBJECT CLASSIFICATION}

E.g., Mathematics Subject Classification; 05C05; 05C12; $92 \mathrm{E} 10$.

\section{Council for Innovative Research}

Peer Review Research Publishing System

Journal: Journal of Advances in Chemistry

Vol 3, No. 1

editor@cirworld.com

www.cirworld.com, member.cirworld.com 


\section{INTRODUCTION}

Let $G=(V ; E)$ be a simple connected graph of finite order $n=/ V /$ and the number of edges $e=|E|$, such that it has vertex set $V=V(G)$ and edge set $E=E(G)$. A general reference for the notation in graph theory is [1-3]. A molecular graph is a simple finite graph such that its vertices correspond to the atoms and the edges to the bonds.

Mathematical chemistry is a branch of theoretical chemistry for discussion and prediction of the molecular structure using mathematical methods without necessarily referring to quantum mechanics. Chemical graph theory is a branch of mathematical chemistry which applies graph theory to mathematical modeling of chemical phenomena. This theory has an important effect on the development of the chemical sciences.

In chemical graph theory, we have many different topological index of arbitrary molecular graph $G$. A topological index of a graph is a number related to a graph which is invariant under graph automorphisms. Obviously, every topological index defines a counting polynomial and vice versa. The simplest topological indices are the number of vertices and edges of the graph $G$.

Also, an important terminology of graph theory is degree of a vertex $v \in V(G)$, that it is the number of adjacent vertices with $v$ and we denoted by $d_{v}$ (In other words, the degree of a vertex $v$ is equal to the number of its first neighbors.). If $u, v \in V(G)$ then the distance $d_{G}(u, v)$ (or $d(u, v)$ for short) between $u$ and $v$ is defined as the length of (number of edges in) any shortest path in $G$ connecting $u$ and $v$. An edge $e=u v$ of the graph $G$ is joined between two vertices $u$ and $v(d(u, v)=1)$.

The Wiener index $W(G)[4-9]$ is the first reported distance based topological index which have very chemical applications, mathematical properties and is defined as half sum of the distances between all the pairs of vertices in a molecular graph, which:

$$
W \mathrm{G}=\frac{1}{2} \sum_{v \in V(G)} \sum_{u \in V(G)} d u, v
$$

One of important connectivity topological indices is geometric-arithmetic index of $G$. A class of geometric-arithmetic topological indices [10] may be defined as

$$
G A_{\text {general }} G=\sum_{u v \in E(G)} \frac{2 \sqrt{Q_{v} Q_{u}}}{Q_{v}+Q_{u}}
$$

where $Q_{v}$ is some quantity that in a unique manner can be associated with the vertex $v$ of the graph $G$.

The first member of this class for $Q_{v}=d_{v}$ was considered by Vukicevic and Furtula [11], in 2009, and $G A_{1}$ index was defined as

$$
G A_{1} G=\sum_{u v \in E(G)} \frac{2 \sqrt{d_{v} d_{u}}}{d_{v}+d_{u}}
$$

in which degree of vertex $u$ denoted by $d_{G}(u)$ (or $d_{u}$ for short).

The second member of this class was considered by Fath-Tabar et al. [12] by setting $Q_{u}$ to be the number $n_{u}$ of vertices of $G$ lying closer to the vertex $u$ than to the vertex $v$ for the edge $u v$ of the graph $G$ :

$$
G A_{2} \quad G=\sum_{u v \in E(G)} \frac{2 \sqrt{n_{v} n_{u}}}{n_{v}+n_{u}}
$$

The third member of this class was considered by Bo Zhou et al.[13] by setting $Q_{u}$ to be the number $m_{u}$ of edges of $G$ lying closer to the vertex $u$ than to the vertex $v$ for the edge $u v$ of the graph $G$ :

$$
G A_{3} G=\sum_{u v \in E(G)} \frac{2 \sqrt{m_{v} m_{u}}}{m_{v}+m_{u}}
$$

The fourth member of this class was considered by Ghorbani et al.[14] in 2010 as follows:

where $\varepsilon_{u}$ is the number of the eccentricity of vertex $u$.

$$
G A_{4} \quad G=\sum_{u v \in E(G)} \frac{2 \sqrt{\varepsilon_{v} \varepsilon_{u}}}{\varepsilon_{v}+\varepsilon_{u}}
$$

A new member of the class of geometric-arithmetic topological indices was considered by $A$. Graovac et al [15] recently, by setting $Q_{v}$ to be the summation $S_{v}$ of degrees of all neighbors of vertex $v$ in $G S_{v}=\sum_{u v E(G)} d_{u}$ :

$$
G A_{5} G=\sum_{u v \in E(G)} \frac{2 \sqrt{S_{v} S_{u}}}{S_{v}+S_{u}}
$$


Recently, A. Iranmanesh et al introduced [16] the edge version of geometric-arithmetic index on the ground of the endvertex degree $d_{e}$ and $d_{f}$ of edges $e$ and $f$ in a line graph of $G$ as follows

$$
G A_{e}(G)=\sum_{e f \in E(L(G))} \frac{2 \sqrt{d_{e} d_{f}}}{d_{e}+d_{f}}
$$

where the line graph $L(G)$ of a graph $G$ is defined to be the graph whose vertices are the edges of $G$, with two vertices being adjacent if the corresponding edges share a vertex in $G$.

In Refs [17-25] some connectivity and geometric-arithmetic topological indices of some nanotubes and nanotorus are computed. Here our notations are standard and mainly taken from standard books of chemical graph theory [1-3].

\section{Main Results and Discussions}

The goal of this paper is to compute a closed formula of this new Connectivity index "fifth geometric-arithmetic index $G A_{5}$ " of circumcoronene homologous series of benzenoid $H_{k}(k \geq 1)$.

The circumcoronene homologous series of benzenoid is family of molecular graph, which consist several copy of benzene $C_{6}$ on circumference. The first terms of this series are $H_{1}=$ benzene, $H_{2}=$ coronene, $H_{3}=$ circumcoronene, $H_{4}=$ circumcircumcoronene, see Figure 1 and Figure 2, where they are shown. Readers can see a general represetation of $H_{k}$ in Figure 2. In addition, this benzenoid molecular graph is presented in many papers, for further study and more historical details, readers can see the paper series [21-41].

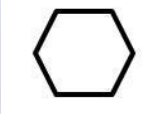

$$
H_{1}=\mathbf{C}_{6}
$$$$
\text { (=benzene) }
$$

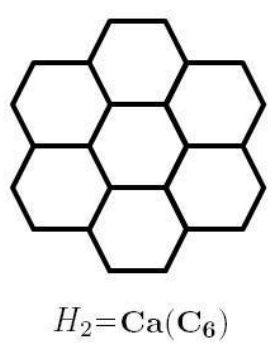

(=coronene)

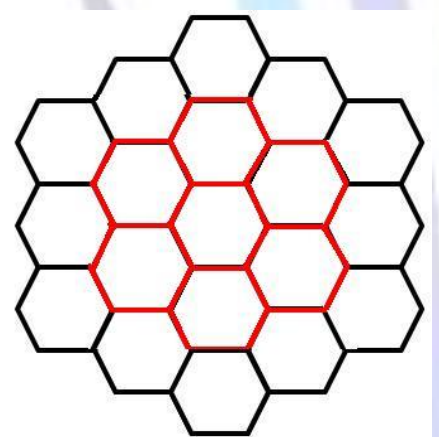

$\mathrm{H}_{3}=$ circumcoronene

Fig. 1. The first three graphs $H_{1}, H_{2}$ and $H_{3}$ from the circumcoronene series of benzenoid [21].

By the above terminologies, we have following theorems. These theorems are main result in this paper.

Theorem 1. [21] Consider the graph $G=H_{k}(k \geq 2)$ is circumcoronene series of benzenoid. Then the Geometric-Arithmetic index $G A\left(H_{k}\right)$ is

$$
G A\left(H_{k}\right)=9 k^{2}+\left(\frac{24 \sqrt{6}}{5}-15\right) k+\left(12-\frac{24 \sqrt{6}}{5}\right)
$$

Theorem 2. [22] Let $G$ be the circumcoronene series of benzenoid $H_{k}(k \geq 1)$. Then Second geometric-arithmetic index $G A_{2}$ of $G$ is equal to

$$
G A_{2}\left(H_{k}\right)=\frac{12}{n_{k}}\left(\sum_{i=1}^{k-1}(k+i) \sqrt{2 k^{2} i^{2}+12 k^{3} i-4 k i^{3}-i^{4}}\right)+6 k
$$

Theorem 3. [23] The third geometric-arithmetic index of circumcoronene series of benzenoid $H_{k}(k \geq 1)$ is equal to

$$
G A_{3} H_{k}=6 k+6 \sum_{i=1}^{k-1} \frac{k+i}{9 k^{2}-4 k-i} \sqrt{\frac{-9}{4} i^{4}-9 k-3 i^{3}+\left(\frac{18 k^{2}+30 k-3}{4}\right) i^{2}+\left(\frac{54 k^{3}-39 k^{2}-k}{2}\right) i-9 k^{3}+3 k^{2}} .
$$

Theorem 4. [24] Let $G$ be the circumcoronene series of benzenoid $H_{k}(\forall k \geq 1)$. Then the eccentric geometric-arithmetic index $G A_{4}$ of $H_{k}$ is equal to 


$$
G A_{4}\left(H_{k}\right)=\sum_{i=2}^{k} 12(i-1)\left(\frac{2 \sqrt{4 i^{2}+8 k-6 i+4 k^{2}-6 k+2}}{4 i+4 k-3}+\frac{\sqrt{4 i^{2}+8 k-10 i+4 k^{2}-10 k+6}}{4 i+4 k-5}\right)+6 k
$$

Theorem 5. [25] For the graphs from the circumcoronene series of benzenoid $H_{k} \forall k \geq 1$

$$
G A_{e}\left(H_{k}\right)=6 k^{2}(3 k-4)+\frac{48 \sqrt{k-1}}{7}+\frac{24 \sqrt{6}}{5}
$$

Theorem 6. Consider the graph $G=H_{k}(k \geq 1)$ is circumcoronene series of benzenoid. Then

$$
G A_{5}\left(H_{k}\right)=9 k^{2}+\left(\frac{24 \sqrt{6}}{5}-15\right) k+\left(12-\frac{24 \sqrt{6}}{5}\right)
$$

Proof. Let $G$ be the circumcoronene series of benzenoid $H_{k}$ for all integer number $k \geq 1$ (Figure 2). The number of vertices/atoms in this benzenoid molecular graph is equal to $\left|V\left(H_{k}\right)\right|=6 k^{2}$ and the number of vertices as degrees 2 and 3 are equal to $\left|V_{2}\right|=6 k$ and $\left|V_{3}\right|=6 k(k-1)$, (we denote $V_{i}:=\left\{v \in V \quad G \mid d_{i}=i\right\}$ ) thus obviously the number of edges/bonds of $G$ is $\left|E\left(H_{k}\right)\right|=\frac{3 \times 6 k k-1+2 \times 6 k}{2}=9 k^{2}-3 k$. Also, it is easy to see that the edge set of $H_{k}$ can be divide in to three partitions, e.g. $E_{4}, E_{5}$ and $E_{6}$ as follow:

- For every $e_{6}=u v$ belong to $E_{6}, d_{v}=d_{u}=3$.

- For every $e_{5}=x y$ belong to $E_{5}$, then $d_{x}=2$ and $d_{y}=3$.

- For every $e_{4}=a b$ belong to $E_{4}$, then $d_{a}=d_{b}=2$.

From Figure 2, we mark the members of $E_{4}, E_{5}$ and $E_{6}$ by red, green and black color and obviously the size of these three edge types are equal to $6,12(k-1)$ and $9 k^{2}-15 k+6$, respectively.

According to Figure 2, one can see that the summation of degrees of vertices of this benzenoid graph have four types, such that for vertices $a, b \in V_{2} \& a b \in E_{4} S(a)=d_{b}+3=5\left(S(b)=d_{a}+3\right)$. And also, for vertices $x \in V_{2} \& y_{1}, y_{2} \in V_{3}$ and edges $x y_{1}, x y_{2} \in E_{5} S(x)=d_{y 1}+d_{y 2}=6$ and $S\left(y_{i}\right)=2+2+3=7$. It is easy to see that for all other vertices $u, v$ from $V_{3}$ and all other edges $e=u v$ belong to $E_{6} S(u)=d_{v}+3+3=9\left(S(v)=d_{u}+3+3\right)$.

Now, by arrangement above formula, we have:

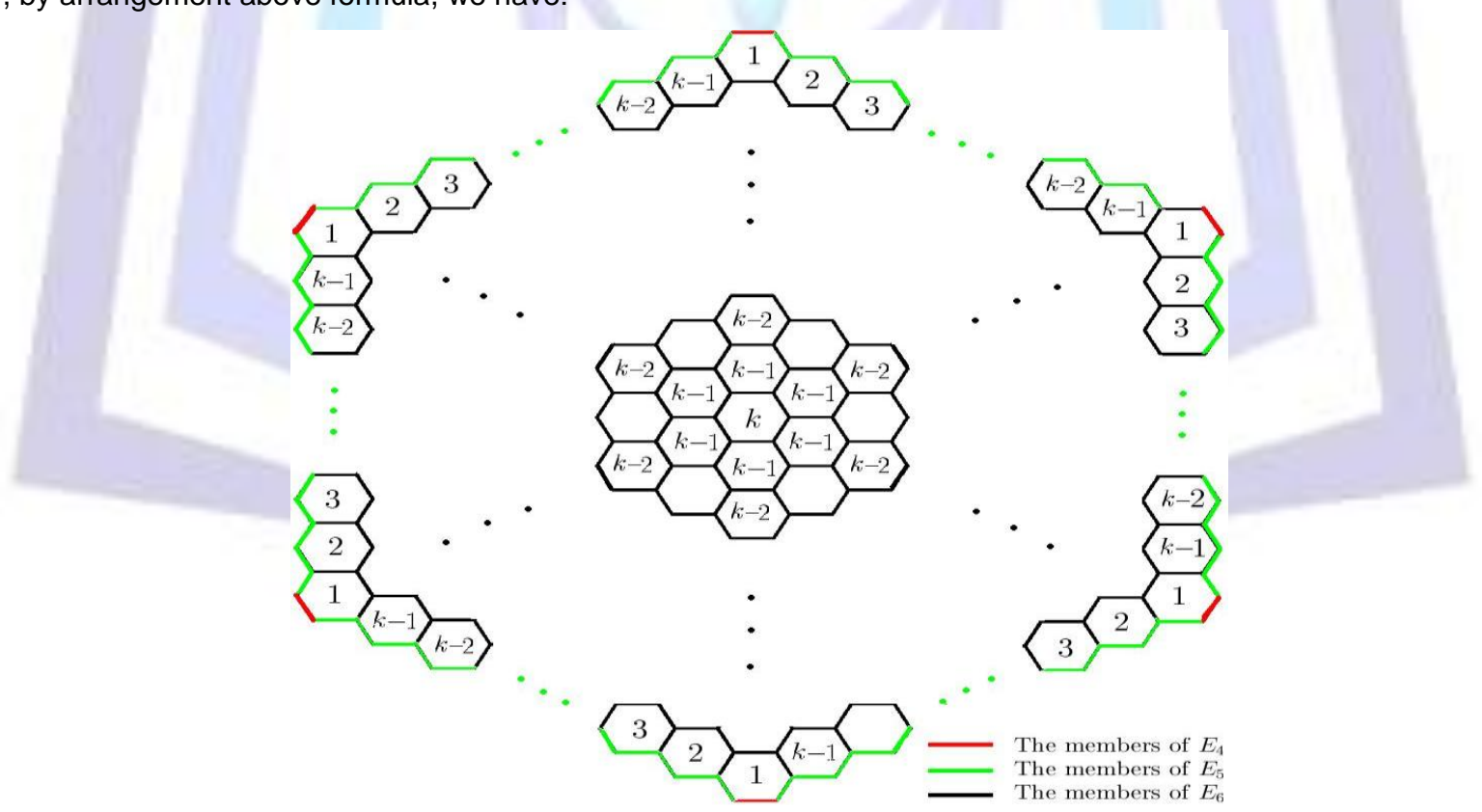

Fig. 2. The circumcoronene series of benzenoid $H_{k}, k \geq 1$, with edges marking [21].

$$
\begin{aligned}
G A_{5}\left(H_{k}\right) & =\sum_{\mathrm{e}=\mathrm{uv} \in E\left(H_{k}\right)} \frac{2 \sqrt{S v S u}}{S v+S u} \\
& =6 \frac{2 \sqrt{5 \times 5}}{5+5}+6 \frac{2 \sqrt{5 \times 7}}{5+7}+2 \times 6 k-2 \frac{2 \sqrt{6 \times 7}}{6+7}+6 k-1 \frac{2 \sqrt{7 \times 9}}{7+9}+\left|E H_{k-1}\right| \frac{2 \sqrt{9 \times 9}}{9+9}
\end{aligned}
$$




$$
=6+\sqrt{35}+\frac{24 k-2 \sqrt{42}}{13}+\frac{9 k-1 \sqrt{7}}{4}+9 k^{2}-21 k+12
$$

Finally, Fifth Geometric-Arithmetic index of circumcoronene series of benzenoid $H_{k}$ is equal to

$$
G A_{5}\left(H_{k}\right)=9 k^{2}+\left(\frac{24 \sqrt{42}}{13}+\frac{9 \sqrt{7}}{4}-21\right)+\left(18+\sqrt{35}-\frac{48 \sqrt{42}}{13}-\frac{9 \sqrt{7}}{4}\right) \text {. }
$$

Here, we complete the proof of Theorem 6.

\section{REFERENCES}

[1] D.B. West. An Introduction to Graph Theory. Prentice-Hall. (1996).

[2] N. Trinajstić. Chemical Graph Theory. CRC Press, Bo ca Raton, FL. (1992).

[3] R. Todeschini and V. Consonni. Handbook of Molecular Descriptors. Wiley, Weinheim. (2000).

[4] H. Wiener, Structural Determination of Paraffin Boiling Points. J. Am. Chem. Soc. 69, 17. (1947).

[5] A.A. Dobrynin, R. Entringer and I. Gutman, Wiener index of trees: Theory and applications, Acta Appl. Math. 66, 211249, (2001).

[6] D.E. Needham, I.C. Wei and P.G. Seybold. Molecular modeling of the physical properties of alkanes. J. Am. Chem. Soc. 110, 4186. (1988).

[7] G. Rucker and C. Rucker. On topological indices, boiling points, and cycloalkanes. J. Chem. Inf. Comput. Sci. 39, 788. (1999).

[8] W. C. Shiu and P. C. B. Lam. The Wiener number of a hexagonal net. Discrete Appl. Math. 73, 101-111. (1997).

[9] B. Zhou and I. Gutman. Relations between Wiener, Hyper-Wiener and Zagreb Indices. Chemical Physics Letters. 394, 93-95. (2004).

[10] B. Furtula, A. Graovac and D. Vukičević, Atom-bond connectivity index of trees, Disc. Appl. Math., 157 (2009), 2828 2835.

[11] D. Vukičević and B. Furtula, Topological index based on the ratios of geometrical and arithmetical means of endvertex degrees of edges, J. Math. Chem., 46 (2009), $1369-1376$.

[12] GH. Fath-Tabar, B. Furtula and I. Gutman, A new geometric--arithmetic index, J. Math. Chem., 47 (2010), 477 - 486.

[13] B. Zhou, I. Gutman, B. Furtula and Z. Du, On two types of geometric-arithmetic index, Chem. Phys. Lett., 482 (2009), $153-155$.

[14] M. Ghorbani and A. Khaki, A note on the fourth version of geometric-arithmetic index, Optoelectron. Adv. Mater. Rapid Comm., 4(12) (2010), 2212-2215.

[15] A. Graovac, M. Ghorbani and M. A. HosseinZadeh. Computing fifth geometric-arithmetic index for nanostar dendrimers, Journal of Mathematical NanoScience, 1(1), 2011, 33-42.

[16] A. Mahmiani, O. Khormali and A. Iranmanesh. On the Edge Version of Geometric-Arithmetic Index, Digest J. Nanomate, Bios., 7(2)., 411-414. (2012).

[17] M.R. Farahani. Computing some connectivity indices of Nanotubes. Advances in Materials and Corrosion 1(2012) 5760.

[18] M.R. Farahani. Fifth Geometric-Arithmetic Index of $T U R C_{4} C_{8}(S)$ Nanotubes. Journal of Chemica Acta. 2(1) (2013).

[19] M. Ghorbani and M. Ghazi. Computing Some Topological Indices of Triangular Benzenoid. Digest. J. Nanomater. Bios. 5, (4), 1107-1111. (2010).

[20] L. Xiao, S. Chen, Z. Guo and Q. Chen. The Geometric-Arithmetic Index of Benzenoid Systems and Phenylenes Int. J. Contemp. Math. Sciences. 5, (45), 2225-2230, (2010).

[21] M.R. Farahani. Computing Randic, Geometric-Arithmetic and Atom-Bond Connectivity indices of Circumcoronene Series of Benzenoid. Int. J. Chem. Model. 5(4), In press (2013).

[22] M.R. Farahani. $G A_{2}$ index of Circumcoronene Series of Benzenoid $H_{k}$. Submitted for publish, 2013.

[23] M.R. Farahani. Using the Cut Method to Computing $G A_{3}$ of Circumcoronene Series of Benzenoid $H_{k}$. Int $J$ Chem Model. 5(2) in press (2013).

[24] M.R. Farahani. Computing a New Connectivity Index for a Famous Molecular Graph of Benzenoid Family. Journal of Chemica Acta, 2, (2013) 26-31.

[25] M.R. Farahani. The Edge Version of Geometric-Arithmetic Index of Benzenoid Graph. Submitted for publish, (2012).

[26] J. Brunvoll, B. N. Cyvin and S.J. Cyvin. Enumeration and Classification of Benzenoid Hydrocarbons. Symmetry and Regular Hexagonal Benzenoids. J. Chem. Inf. Comput. Sci. 27, 171-177. (1987). 
[27] V. Chepoi and S. Klavžar. Distances in benzenoid systems: Further developments. Discrete Mathematics. 192, 27-39. (1998).

[28] J.R. Dias. From benzenoid hydrocarbons to fullerene carbons. MATCH Commun. Math. Comput. Chem. 4, 57-85. (1996).

[29] M.V. Diudea. Studia Univ. Babes-Bolyai. 4, 3-21. (2003).

[30] A. Dress and G. Brinkmann. Phantasmagorical fulleroids, MATCH Commun. Math. Comput. Chem. 33, 87-100. (1996).

[31] M.R. Farahani and M.P. Vlad. On the Schultz, Modified Schultz and Hosoya polynomials and Derived Indices of Capra-designed planar Benzenoids. Studia Univ. Babes-Bolyai. 57(4) In press, (2012).

[32] M.R. Farahani. On the Schultz polynomial, Modified Schultz polynomial, Hosoya polynomial and Wiener index of circumcoronene series of benzenoid, J. Applied Mathe. \& Informatics. 31(3) in press, (2013).

[33] M.R. Farahani. Zagreb index, Zagreb Polynomial of Circumcoronene Series of Benzenoid. Advances in Materials and Corrosion. 2 (2013) 16-19

[34] M. Goldberg. A class of multi-symmetric polyhedra. Tohoku Math. J. 43, 104-108. (1937).

[35] A. Ilic, S. Klavžar and D. Stevanovic. Calculating the Degree Distance of Partial Hamming Graphs. MATCH Commun. Math. Comput. Chem. 63, 411-424, (2010).

[36] S. Klavžar and I. Gutman. Bounds for The Schultz Molecular Topological Index of benzenoid Systems in Terms of Wiener Index. J. Chem. Inf. Comput. Sci. 37, (4), 741-744. (1997).

[37] S. Klavžar. A Bird's Eye View of The Cut Method and a Survey of Its Applications in Chemical Graph Theory. MATCH Commun. Math. Comput. Chem. 60, 255-274. (2008).

[38] S. Klavžar, I. Gutman and B. Mohar. Labeling of Benzenoid Systems which Reflects the Vertex-Distance Relations. J. Chem. Inf. Comput. Sci. 35, 590-593. (1995).

[39] S. Klavžar and I. Gutman. A Comparison of the Schultz Molecular Topological Index with the Wiener Index. J. Chem. Inf. Comput. Sci. 36, 1001-1003. (1996).

[40] K. Salem, S. Klavžar and I. Gutman. On the role of hypercubes in the resonance graphs of benzenoid graphs. Discrete Mathematics. 13(8) 306, (2003).

[41] A. Soncini, E. Steiner, P.W. Fowler, R.W.A. Havenith, and L.W. Jenneskens. Perimeter Effects on Ring Currents in Polycyclic Aromatic Hydrocarbons, Circumcoronene and Two Hexabenzocoronenes. Chem. Eur. J. 9, $2974-2981$. (2003). 